\title{
Relapses During High-Dose Biotin Treatment in Progressive Multiple Sclerosis: a Case-Crossover and Propensity Score-Adjusted Prospective Cohort
}

\author{
Pierre Branger $^{1}$ (D) Jean-Jacques Parienti ${ }^{2} \cdot$ Nathalie Derache $^{1} \cdot$ Nizam Kassis $^{3} \cdot$ Rana Assouad $^{4} \cdot$ Elisabeth Maillart $^{4}$. \\ Gilles Defer ${ }^{1}$
}

Published online: 15 June 2020

(C) The American Society for Experimental NeuroTherapeutics, Inc. 2020

\begin{abstract}
High-dose biotin (HDB) is a therapy used in non-active progressive multiple sclerosis (PMS). Some reports have suggested that HDB treatment may be associated with an increased risk of relapse. We evaluate the relationship between exposure to HDB for treating PMS and the risk of relapse. We screened for PMS patients prospectively registered in a French regional cohort being part of the OFSEP national registry. In a case-crossover design among patients who received HDB, we first compared number of relapses before and after initiation of HDB. Second, time to the first clinical relapse was compared between patients who received HDB (biotin group) and a control group using a Cox survival analysis after a propensity score (PS) matching (1:1) and inverse probability of treatment weighting (IPTW) method. In the 42 PMS patients who received HDB, the number of relapses was statistically and clinically significant higher after biotin initiation than before biotin initiation (incident rate ratio [IRR] 7.4, 95\% confidence interval [CI] 3.5$15.9, p<0.0001)$. With the PS matching method, the risk of relapse was significantly higher in the biotin group compared to the control group (hazard ratio [HR] 4.3, 95\% CI 1.4-13.3, $p=0.01$ ). The IPTW method with 440 control patients revealed consistent results (HR 5.1, 95\% CI 2.3-11.3, $p<0.0001$ ). In our non-randomized study, HDB treatment for PMS was associated with an increased risk of relapse. The follow-up of PMS patients initiating HDB should include careful assessment of clinical and radiological activity to monitor the potential pro-inflammatory effect of biotin.
\end{abstract}

Key Words Multiple sclerosis $\cdot$ relapses $\cdot$ biotin $\cdot$ progressive $\cdot$ propensity score

\begin{abstract}
Abbreviations
HDB High-dose biotin

MS Multiple sclerosis
\end{abstract}

Electronic supplementary material The online version of this article (https://doi.org/10.1007/s13311-020-00880-z) contains supplementary material, which is available to authorized users.

Pierre Branger

branger-p@chu-caen.fr

Department of Neurology, CHU de Caen, F-14000 Caen, France

2 Department of Clinical Research and Biostatistics, CHU de Caen, F-14000 Caen, France

3 Department of Neurology, CHP du Cotentin, F-50102 Cherbourg-en-Cotentin, France

4 Department of Neurology, GH Pitié-Salpêtrière, F-75013 Paris VI, France
PMS Progressive multiple sclerosis

ARR Annualized relapse rate

PS Propensity score

IPWT Inverse probability of treatment weighting

\section{Introduction}

Progressive multiple sclerosis (PMS) can be categorized as active or non-active depending inflammatory activity [1]. New therapies have recently demonstrated some efficacy in PMS. Ocrelizumab was associated with lower rates of clinical and MRI progression than placebo [2] and high-dose biotin (HDB)-treated patients achieved sustained reversal of MSrelated disability in non-active PMS [3]. In this study, more new or enlarging MRI lesions occurred in the treated arm than in the placebo arm (23.4\% vs $13.0 \%$ respectively, $p=0.36)$ with more relapses in the extension phase $(6.6 \%$ in patients 
who initially received HDB and $4.8 \%$ in patients who initially received placebo). Since this pilot trial, some reports have suggested that HDB treatment may be associated with an increased risk of relapse in PMS patients with previously stable disease $[4,5]$.

As we also observed unexpected moderate to severe relapse in PMS patients who had very stable disease without clinical or radiological activity before being treated with HDB, we decided to evaluate the relationship between exposure to HDB treatment and the risk of relapse by analyzing all PMS cases from our regional MS cohort. Additional case reports were submitted to our advice from other MS expert centers that were briefly reported in 2018 [6].

\section{Methods}

\section{Study Population}

In the regional cohort of Caen MS center in France, being part of the OFSEP national registry [7], 729 PMS patients were identified in December 2017. Age, sex, PMS form (primary or secondary PMS), EDSS, treatments, date of progression, and relapses were collected. All patients under HDB received $300 \mathrm{mg}$ of biotin (100 mg thrice a day). Relapses were reported by the neurologists of our MS expert center (PB, ND, GD) according to the international definition of relapse. The start of follow-up period was established in the same period of natural history of MS in all patients: after biotin initiation in biotinexposed patients (mean duration was 103 months between progression onset and biotin initiation) and 103 months after progression onset in non-biotin exposed patients (Fig. 1). We excluded patients with relapse in the previous year of the follow-up period, patients without data after onset of follow- up period, patients without EDSS at progression, and patients without data before initiation of HDB.

\section{Standard Protocol Approvals, Registrations, and Patient Consents}

All patients gave informed consent for their data to be stored in the database and used for research. Data confidentiality and safety are ensured according to the recommendations of the French Commission Nationale Informatique et Libertés (CNIL). The cohort has been registered to clinicaltrials.gov under the number NCT02889965.

\section{Statistical Analyses}

First, in a case-crossover design among patients who received HDB, we defined the following: (i) the period before HDB initiation as the time between the onset of progression phase of multiple sclerosis and HDB initiation and (ii) the period after HDB initiation as the time between HDB initiation and last observation with HDB. We compared the number of relapses before and after the initiation of HDB using a generalized linear model with Poisson distribution, exchangeable correlation structure, and the log of the period as an offset.

Second, regarding the propensity score methods, two approaches were used, namely propensity score (PS) matching and inverse probability of treatment weighting (IPWT). The propensity score, which represents the probability of being exposed to biotin conditional to baseline characteristics, was computed by non-parsimonious logistic regression model including age at progression, sex, primary PMS form, and EDSS at progression. In survival analyses, patients follow-up was censored after 12-month. Patients exposed to biotin were 1:1 matched to controls using the greedy matching procedure based on the PS. The balance between groups after PS matching was checked by
Fig. 1 Estimation of follow-up period in survival analyses. In the biotin group, the start of followup period began after biotin initiation. In the control group, the start of follow-up began 103 months after progression onset, corresponding at mean duration between progression onset and biotin initiation in the biotin group, to be in the same period of natural history of MS in all patients

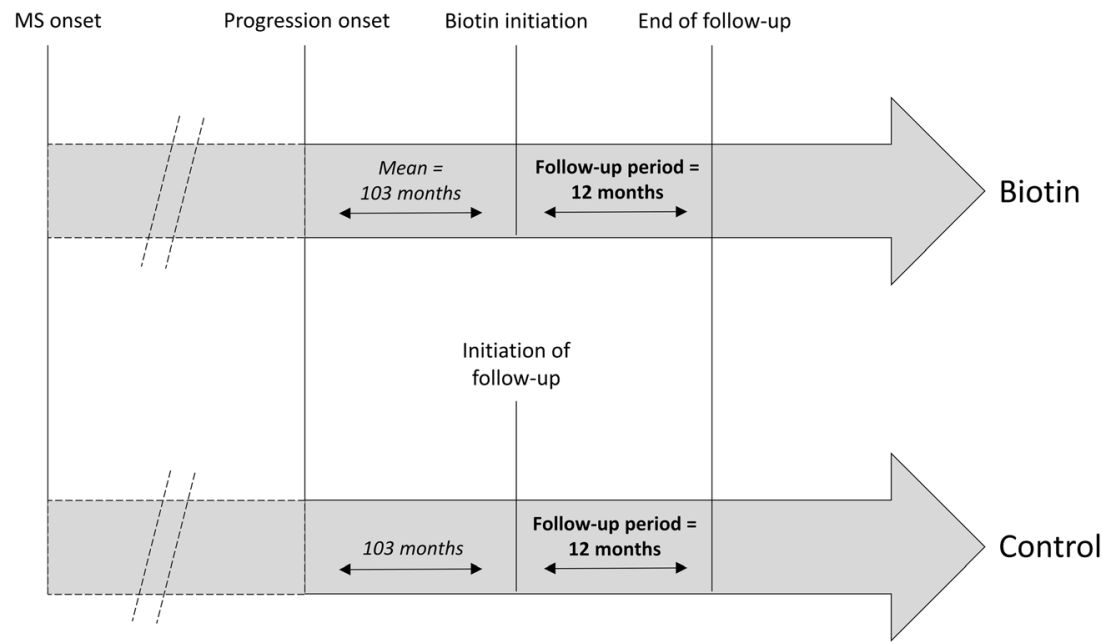


computing the standardized difference, with a value below $10 \%$ indicating similar baseline characteristics. Time to relapse was compared between biotin and control groups by a Cox model using robust covariance matrix estimation to account for the paired design. In addition, we conducted an IPWT analysis using a Cox model with stabilized weights to compare the time to relapse in the overall cohort. A sensitivity analysis including concomitant DMD at the start of follow-up in the PS was conducted. All analyses were conducted with SAS 9.4 (SAS institute, $\mathrm{NC}$, Cary) and a $p$ value $<0.05$ was considered to denote statistical significance.

\section{Data Availability}

Because of the confidentiality and security concerns, only applicants with an OFSEP-approved project and the members of the project teams are allowed to have direct access to the raw data.

\section{Results}

We identified 729 PMS patients in our regional OFSEP database and 482 PMS patients were included (mean age $60.0 \pm$ 9.4 years, $62 \%$ female, $50 \%$ primary PMS, mean EDSS $6.6 \pm$ 1.7). Forty-two PMS patients received HDB and 440 did not received biotin (fig. 2). Eleven patients (26.2\%) developed relapses during biotin treatment. Four patients (9.5\%) stopped HDB, one after a relapse. Characteristics of patients are described in Table 1. First, in the case-crossover analysis among patients who received $\mathrm{HDB}$, the annualized relapse rate (ARR) ratio was $0.04 \pm 0.08$ in the progressive phase before biotin and $0.39 \pm 0.77$ after biotin. Using the Poisson regression model, the number of relapses was statistically and clinically significantly higher after biotin initiation than before biotin initiation (incident rate ratio [IRR] 7.4, 95\% confidence interval [CI] 3.5-15.9, $p<0.0001)$.

Second, with the PS matching method, the risk to develop a relapse was significantly higher in the biotin group compared to the control group (hazard ratio [HR] 4.3, 95\% CI 1.4-13.3, $p=0.01$; Fig. 3). The standardized difference between groups after PS matching was below $10 \%$ for all the baseline variables included in the PS. Using the IPTW method, result was consistent with higher risk in biotin group (HR 5.1, 95\% CI 2.3-11.3, $p<0.0001$, Fig. 4). A sensitivity analysis including patients with concomitant DMD at the start of follow-up in the PS was consistent with our previous results (HR 5.56, 95\% CI 1.6-19.7, $p=0.008$ with PS matching method and HR 4.29, 95\% CI 1.8-10.0, $p=0.0007$ with IPTW method).

\section{Discussion}

In this regional cohort of PMS patients, HDB treatment is associated with a higher risk of relapse, using three different statistical methods. Obviously, we observed at a higher rate a shift from a main neurodegenerative status towards an unexpected clinical and radiological activity suggesting a potential pro-inflammatory effect of this treatment. Even if clinical or radiological activity were also present in PMS without
Fig. 2 Patient disposition. PMS = Progressive multiple sclerosis, EDSS $=$ Expanded disability status scale, PSM = Propensity score matching, IPTW = Inverse probability of treatment weighting

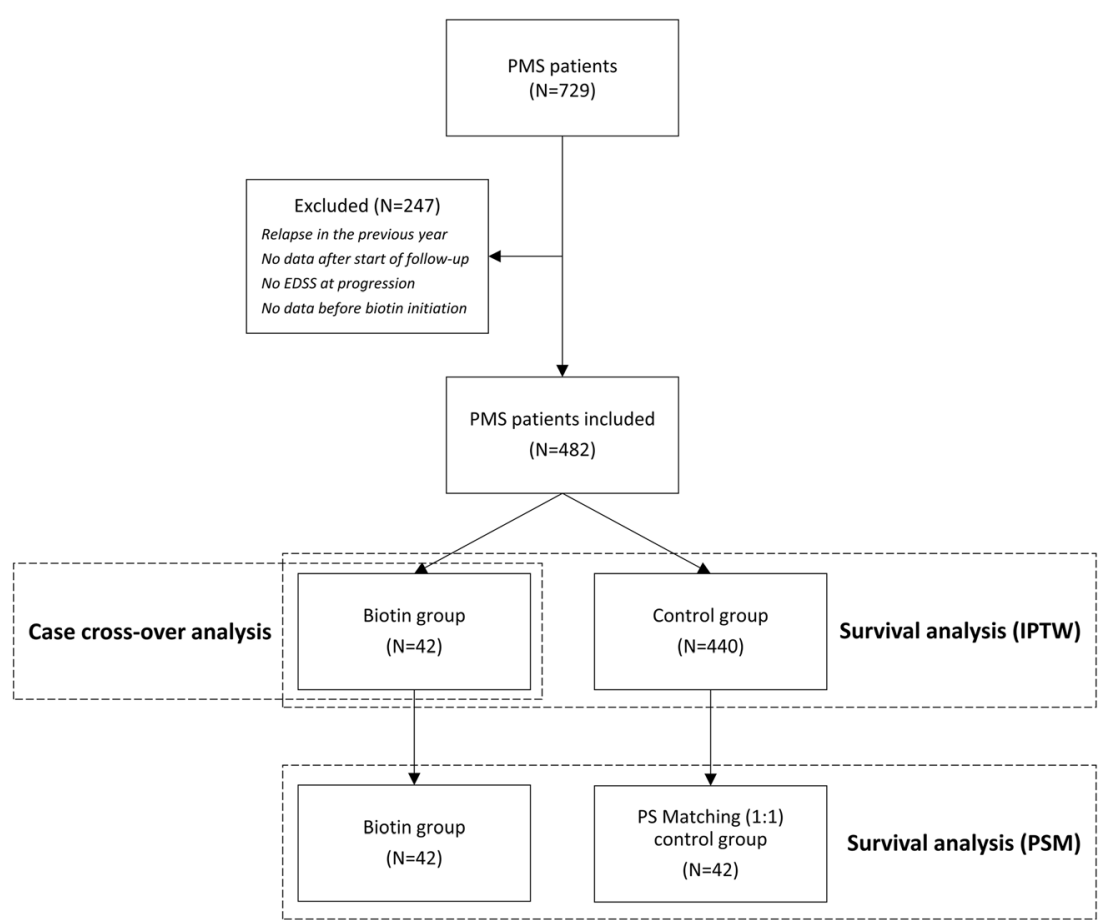


Table 1 Characteristics of included patients

\begin{tabular}{lll}
\hline & Biotin group* $(N=42)$ & Control group $(N=440)$ \\
\hline Women, $N(\%)$ & $22(52.4)$ & $279(63.4)$ \\
Primary PMS form, $N(\%)$ & $21(50.0)$ & $218(49.5)$ \\
Age at progression onset, year & $48.1(8.6)$ & $42.4(10.0)$ \\
EDSS at progression onset & $4.5(1.7)$ & $5.5(2.1)$ \\
MS duration at progression onset, year & $7.7(9.6)$ & $6.3(8.6)$ \\
Concomitant DMD, $N(\%)$ & & $36(8.2)$ \\
Patients who stopped DMD during the year before follow-up & $4(9.5)$ & $125(28.4)$ \\
Patients with concomitant DMD at the start of follow-up & $6(14.3)$ & $67(17.5)$ \\
$\quad$ Immunosuppressive DMD & $3(7.1)$ & $45(10.2)$ \\
$\quad$ First-line DMD & $2(4.8)$ & $3(0.7)$ \\
$\quad$ Other & $1(2.4)$ & $127(28.9)$ \\
Patients with concomitant DMD at the end of follow-up & $5(11.9)$ & \\
\hline
\end{tabular}

Unless otherwise indicated, data are expressed as means (SD)

EDSS $=$ Expanded disability status scale, DMD = Disease-modifying drugs

*All patients in the biotin group received $300 \mathrm{mg}$ of biotin (100 $\mathrm{mg}$ thrice a day)

${ }^{\dagger}$ Including mitoxantrone, cyclophosphamide, methotrexate, azathioprine, mycophenolate mofetil, and methylprednisolone

${ }^{*}$ Including interferons, glatiramer acetate, and dimethyl fumarate

treatment (3 to $30 \%$ [8]), relapses after recent HDB treatment in non-active MS patients challenge the question of a potential relationship. In the pivotal placebo-controlled study [3], more new or enlarging MRI lesions occurred in the HDB arm than in the placebo arm during the double-blind phase. In the MSON study [9], the incidence of MS relapse was higher in the biotin group than in the placebo group during the double-blind phase of the study and was also higher in the patients newly treated with biotin in the extension phase than in the placebo group in the double-blind phase. A cohort of consecutive PMS patients treated with HDB showed an unexpected high rate of clinical and radiological inflammation (relapses in 9/41 patients, MRI activity in $7 / 41$ patients, ARR after treatment 0.27 vs 0.1 before treatment [4]).

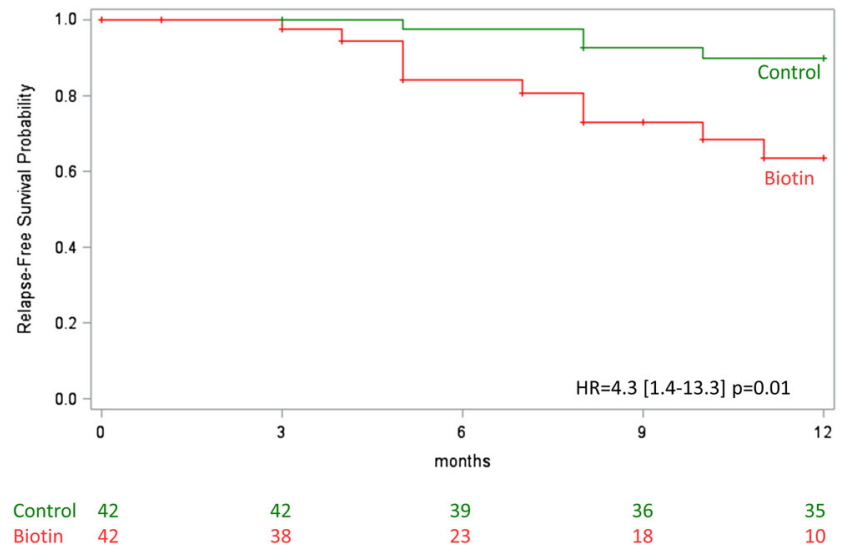

Fig. 3 Time to the first relapse with the PSM method. Cox model using robust covariance matrix estimation to account for the paired design. HR $=$ Hazard ratio
Mechanisms through which biotin may facilitate relapse in PMS has to be elucidated. We can suspect unknown risk factors facilitating relapse in some patients, but analyses included main clinical parameters in the model. The other hypothesis may be metabolic. Indeed, biotin acts as a coenzyme for five carboxylases involved in energy metabolism, fatty acid synthesis, and amino acid catabolism [10,11], and it has been suggested that HDB treatment could reverse a "virtual hypoxia", a mechanism explaining progressive degeneration, caused by a mismatch between increased energy demand by the demyelinated axon and decreased energy production because of mitochondrial injury $[11,12]$. HDB treatment could reverse this state by triggering myelin synthesis by oligodendrocytes and by replenishing ATP in hypoxic neurons. In addition,

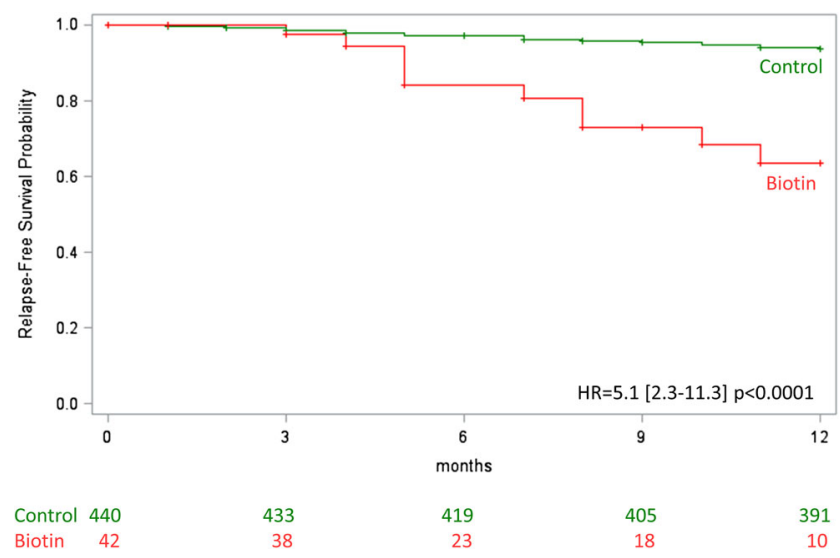

Fig. 4 Time to the first relapse with the IPWT method. Cox model with stabilized weights to compare the time to relapse in the overall cohort. HR = Hazard ratio 
glycolysis and/or mitochondrial ATP production is an essential phenomenon in the metabolic functioning of activated lymphocytes. Augmentation of activity of methylcrotonylCoA carboxylase, a biotin-dependent carboxylase, could increase catabolism of leucine with production of beta-hydroxybeta-methylbutyrate [13], known to increase proliferation of lymphocytes. This remains to be demonstrated by complementary studies.

This study has some limitations. First, our cohort only covered a limited regional area and could not be transposed to a larger population. The number of excluded PMS patients was important due to missing values, mainly in the control group. A French multicenter study evaluating incidence of relapses in patients with HDB-treated progressive MS did not show significant increased risk of clinical inflammatory activity [14] but the statistical analysis used was different. Previous treatments may also affect the risk of relapse but treatments stopped during the year before inclusion were similar in the two groups in our study. The number of patients with concomitant DMD at the start of follow-up was different between patient groups because HDB was introduced generally in patients without previous DMD and not used as an add-on therapy. Another limitation is that the role of MRI activity was not evaluated in our study as an outcome and in the propensity model, because of lack of interpretable MRI data.

Our results suggest follow-up of PMS patient initiating HDB should include careful assessment of inflammatory activity (ARR and MRI). An MRI monitoring may be recommended before and after introduction of biotin in PMS patients to detect new T2 or gadolinium-enhancing lesions suggesting resumption of inflammatory activity.

Required Author Forms Disclosure forms provided by the authors are available with the online version of this article.

\section{Compliance with Ethical Standards}

Disclosures The authors declare that there is no conflict of interest.

\section{References}

1. Lublin FD, Reingold SC, Cohen JA, et al. Defining the clinical course of multiple sclerosis: the 2013 revisions. Neurology 2014;83:278-286.

2. Montalban X, Hauser SL, Kappos L, et al. Ocrelizumab versus placebo in primary progressive multiple sclerosis. N Engl J Med 2017;376:209-220.

3. Tourbah A, Lebrun-Frenay C, Edan G, et al. MD1003 (high-dose biotin) for the treatment of progressive multiple sclerosis: a randomised, double-blind, placebo-controlled study. Mult Scler 2016;22:1719-1731.

4. Granella F, Tsantes E, Siena E, et al. Breakthrough disease under high-dose biotin treatment in progressive multiple sclerosis. Mult Scler 2017;23:85-426 (P750).

5. Lebrun C, Cohen M, Mondot L, et al. A case report of solitary sclerosis: this is really multiple sclerosis. Neurol Ther 2017;6: 259-263.

6. Branger P, Derache N, Kassis N, et al. Relapses during high doses of biotin in progressive multiple sclerosis: a case series (P5.348). Neurology 2018;90(S15).

7. Vukusic S, Casey R, Rollot F, et al. Observatoire Français de la Sclérose en Plaques (OFSEP): a unique multimodal nationwide MS registry in France. Mult Scler 2020;26(1):118-122.

8. Paz Soldán MM, Novotna M, Abou Zeid N, et al. Relapses and disability accumulation in progressive multiple sclerosis. Neurology 2015;84:81-88.

9. Tourbah A, Gout O, Vighetto A, et al. MD1003 (high-dose pharmaceutical-grade biotin) for the treatment of chronic visual loss related to optic neuritis in multiple sclerosis: a randomized, doubleblind, placebo-controlled study. CNS Drugs 2018;32:661-672.

10. Sedel F, Bernard D, Mock DM, et al. Targeting demyelination and virtual hypoxia with high-dose biotin as a treatment for progressive multiple sclerosis. Neuropharmacology 2016;110:644-653.

11. Peyro Saint Paul L, Debruyne D, Bernard D, et al. Pharmacokinetics and pharmacodynamics of MD1003 (highdose biotin) in the treatment of progressive multiple sclerosis. Expert Opin Drug Metab Toxicol 2016;12:327-344.

12. Sedel F, Papeix C, Bellanger A, et al. High doses of biotin in chronic progressive multiple sclerosis: a pilot study. Mult Scler Relat Disord 2015;4:159-169.

13. Lee AJ, Beno DWA, Zhang X, et al. A (14)C-leucine absorption, distribution, metabolism and excretion (ADME) study in adult Sprague-Dawley rat reveals $\beta$-hydroxy- $\beta$-methylbutyrate as a metabolite. Amino Acids 2015;47:917-924.

14. Mathais S, Moisset X, Taithe F, et al. Incidence of Relapses in Patients with Biotin-Treated Progressive Multiple Sclerosis (IPBio-SeP Study): intermediate analysis of a French, multicenter study (P3.2-038). Neurology 2019;92(S15).

Publisher's Note Springer Nature remains neutral with regard to jurisdictional claims in published maps and institutional affiliations. 